\title{
The Orbital Overlap Distance in Graphene Defects
}

\author{
Arshad Mehmood ${ }^{1}$ and Benjamin Janesko ${ }^{1}$ \\ ${ }^{1}$ Texas Christian University
}

May 6, 2020

\begin{abstract}
Quantum-chemical "descriptors", including atomic partial charges, orbitals, and electrostatic potentials are powerful tools for understanding chemical reactivity. Localized defects in graphene are a particular challenge for these tools, especially to model the adsorption processes and to predict the interactions of transition metals with these defects. Such defects often have little charge polarization and a combination of localized and delocalized states. Our orbital overlap distance $\mathrm{D}(\mathbf{r})$ measures the "size" of occupied orbital lobes about point $\mathbf{r}$, distinguishing the hybridization state and compact vs. diffuse character of local electronic structure. Here we apply the overlap distance to graphene defects. We find that the overlap distance clearly distinguishes differential reactivities of different atoms at intrinsic defects. Combining the overlap distance and electrostatic potential provides a rich picture of extrinsic defect reactivity, including semiquantitative predictions of transition metal binding.
\end{abstract}

\section{Hosted file}

Main_document.pdf available at https ://authorea.com/users/318626/articles/448561-the-orbitaloverlap-distance-in-graphene-defects 